\title{
Psychopharmacological adverse effects
}

Cite as: Keogh, B. \& Doyle, L. , Psychopharmacological adverse effects, Mental Health Practice, 11, (6), 2008, p28 - 30

Brian Keogh RPN, BNS, MSc

Lecturer in Mental Health Nursing

School of Nursing \& Midwifery

Trinity College

24 D'Olier Street

Dublin 2

Ireland

Phone - 018963109

Email-keoghbj@tcd.ie

Louise Doyle, RPN, BNS, RNT, MSc

Lecturer in Mental Health Nursing

School of Nursing \& Midwifery

Trinity College

24 D'Olier Street

Dublin 2

Ireland

Phone - 018963102

Email_louise.doyle@tcd.ie 


\section{Introduction}

Recent advances in the care of people with mental health problems have advocated for a move towards psychosocial based interventions and a reduction in the emphasis on medication based treatments. Despite this, psychopharmacology remains the main treatment for the majority of people in mental distress. The role of the mental health nurse in the administration of medications has become increasingly complex given the range of preparations available and their potential to induce adverse and toxic effects in susceptible individuals. Recognising adverse and toxic effects of psychopharmacology is a key part of the mental health nurse's role and involves assisting individuals to manage side effects which may impact on the individuals' quality of life. Occasionally medications used in contemporary psychiatry have the ability to induce potentially life threatening adverse reactions in a small number of individuals. Given the idiosyncratic nature of some of these effects and the difficulty in predicting people who are vulnerable, early recognition and intervention is imperative to a successful outcome should these occur. The purpose of this article is to provide mental health nurses with some guidance on the recognition and management of these potentially fatal adverse effects, namely agranulocytosis, neuroleptic malignant syndrome, serotonin syndrome and lithium toxicity.

\section{Agranulocytosis}

Agranulocytosis is a blood disorder (dyscrasia) which is characterised by a selective reduction in white blood cells, particularly neutrophils, resulting in an increased susceptibility to infection in those affected (Downie et al. 2004). Rosenfeld \& Loose Mitchell (1998) suggest that blood disorders are relatively rare for clients taking anti 
psychotic drugs, except in the case of Clozapine which may induce agranulocytosis in up to $3 \%$ of individuals taking the drug. Clozapine is classed as an atypical anti psychotic drug which is indicated in treatment resistant schizophrenia. Although it's side effect profile is similar to conventional anti psychotics, clozapine's potential to induce agranulocytosis caused its removal from production in the 1970's. It was reintroduced in the 1990s and can only be prescribed under strict supervision by the Clozapine Patient Monitoring Service. Individuals prescribed the drug must have a blood test to eliminate agranulocytosis weekly for the first 18 weeks, fortnightly for up to a year after this initial period and then monthly thereafter (Healy 2005). Agranulocytosis in itself is difficult to detect and is usually detected when symptoms of infection appear. These symptoms include a raised temperature, sore throat and mucosal ulcers. Nurses need to be vigilant of the symptoms in patients taking clozapine and intervene accordingly. When detected early, intervention should only require cessation of the drug and symptomatic treatments such as for a sore throat or raised temperature. People who continue on anti psychotic medication, especially clozapine, after discharge should also be aware of this side effect and what to do if it occurs. Once agranucytosis has been detected, the individual's condition should be monitored closely however; there is spontaneous recovery for most people within about 2 weeks (Downie et al. 2004).

\section{Neuroleptic Malignant Syndrome}

Neuroleptic Malignant Syndrome (NMS) is a potentially life threatening but relatively rare idiosyncratic reaction to neuroleptic medications. It is generally more likely to occur following the administration of high potency/low dose typical antipsychotics such as Haloperidol. However, it can occur in response to any neuroleptic medication and in 
some cases it can occur when the client is receiving anti depressants (Galbraith et al 2007). It also occurs more frequently in those who are on higher doses of antipsychotic medications and where polypharmacy occurs (Healy 2005). Many clients will experience NMS shortly after initial exposure to antipsychotic medications, and almost all clients who develop it do so within two weeks of commencing antipsychotic medications. However, it can also develop in clients who have taken antipsychotics over a long period of time. Diagnosis of NMS can be complicated as it initially presents in a similar way to Serotonin Syndrome (discussed later in the article).

The symptoms associated with NMS include:

- Muscular rigidity known as lead pipe rigidity

- Tremor

- Hyperthermia

- Urinary incontinence

- Altered mental status (e.g. confusion)

- Altered autonomic function (e.g. high or low blood pressure, elevated or rapid pulse)

- Elevated serum creatinine phosphokinase secondary to muscle breakdown

- Elevated white blood cell count

\section{Treatment and Prevention}

Early detection is essential to the prevention of severe NMS however early diagnosis can be made difficult if the cardinal symptom of muscle rigidity is not clearly evident. It is imperative that nurses are familiar with other key symptoms such as altered mental status, 
fever and altered autonomic function. However, these symptoms could be indicative of changes to the client's mental health or could have an alternative medical cause. Immediate discontinuation of the antipsychotic is essential. Pharmacological interventions include the possible use of a dopamine agonist such as Bromocriptine to increase the production of dopamine and/or a muscle relaxant such as Dantrolene (BNF 2007). In conjunction with this, antipyretics such as paracetamol can be given to reduce fever if indicated. Electroconvulsive Therapy (ECT) may produce a rapid response to NMS and may also be beneficial for the underlying psychiatric condition (Pelonero et al. 1998; Healy 2005). Because of the idiosyncratic nature of NMS, prevention can be difficult as it is rarely possible to accurately predict who will develop the syndrome. Pelonero et al. (1998) identify that agitation, dehydration and a prior history of NMS are risk factors to the development of the syndrome. Therefore it is important nurses are familiar with a client's previous reaction to antipsychotic medication and are aware and respond to deficits in fluid balance in clients taking antipsychotic medications.

\section{Nursing Care}

Apart from pharmacological interventions, the main course of treatment for NMS is symptomatic management. A cooling blanket and/or a fan may be required to help reduce pyrexia. Clients with NMS should be cared for in a high observation area as their physical and mental condition needs to be monitored closely. Routine observations should be taken regularly and documented, with anomalies reported to the responsible medical practitioner. The client may need assistance with their activities of daily living as well as careful utilisation of appropriate interpersonal skills such reality reorientation if confusion exists. Intravenous fluids may also be necessitated to correct dehydration and 
electrolyte abnormalities. Attention to nutritional support is required as many clients may not be able to eat/drink due to altered mental status. The syndrome which usually lasts for 5-7 days after drug discontinuation may be unduly prolonged if depot antipsychotics have been used. Where possible, clients with a history of NMS should not be recommenced on antipsychotic therapy and should instead be prescribed alternative medications such as lithium, carbamazepine or benzodiazepines. However, this may not always be possible and in these cases the client should be switched to an antipsychotic in a different class and with a lower D2 affinity (e.g. atypical antipsychotics) than the one which produced the NMS.

\section{Serotonin Syndrome}

Serotonin Syndrome (SS) is a rare but life-threatening drug reaction caused by an excess of serotonin. In the majority of cases it is caused by a build up of serotonin which is caused when drugs which act on the serotonergic system are prescribed with other drugs that also work on this system (e.g. Selective Serotonin Reuptake Inhibitors, (SSRIs), prescribed with Tricyclic medications). It is generally more likely to occur within the first 24 hours after taking the medication or after an increase/overdose in medication. As SSRIs are now the first line treatment for people with depression it is imperative that nurses are familiar with the signs, symptoms and management of this potentially lifethreatening condition.

The symptoms associated with SS include:

- Jerks and twitches (Myoclonus)

- Tremors of the tongue or fingers

- Shivering 
- Raised temperature

- Sweating

- Confusion, agitation or restlessness

- Tachycardia

- Hyperreflexia

- Diarrhoea

- Intoxication and Euphoria (Galbraith et al 2007)

Before a diagnosis is made, at least three major symptoms should be apparent and the client must be taking a medication that affects the serotonergic system. The main differential diagnosis is Neuroleptic Malignant Syndrome as both conditions share many features however the presence of diarrhoea, the more rapid onset of the disorder and the absence of 'lead pipe' rigidity can help to establish a diagnosis of SS (Birmes et al. 2003). In most cases, serotonin syndrome will resolve once the offending agent(s) have been stopped. Supportive care is essential in the management of SS. Intravenous fluids may be required and the nurse should monitor vital signs and urine output regularly. As with NMS, hyperthermia can be managed by introducing measures to reduce the high temperature (e.g. cooling blankets, fans etc.). The client should also be encouraged to drink plenty of fluids. In more severe cases, a serotonin antagonist such as Cyproheptadine may be used to reduce serotonin levels although the beneficial effects of such medications are not firmly established. 


\section{Lithium Toxicity}

Lithium is a commonly used drug in the treatment and prophylaxis of Bi polar affective disorder and depression. It has many adverse effects and contraindications but it's most worrying is lithium toxicity, which can be fatal and should be treated immediately. Lithium is well known for its narrow therapeutic/toxic range and doses are adjusted to a serum lithium level of $0.4-1 \mathrm{mmol} / \mathrm{litre}$ (BNF 2007). Patients can become toxic for a number of reasons; overdose (accidental or purposeful), dehydration, or infections although in certain instances the cause is unclear (Healy 2005). Toxic symptoms can occur at serum levels of $1.5 \mathrm{mmol} / \mathrm{litre}$ and require emergency treatment if levels are $2 \mathrm{mmol} /$ litre or above.

\section{Recognising Lithium Toxicity}

The symptoms associated with lithium toxicity are:

- Nausea and vomiting

- Diarrhoea

- Tremor (Healy 2005)

However in more serious cases symptoms include:

- Hyperflexia

- Hyperextension of limbs

- Convulsions

- Toxic psychoses

- Syncope 
- Renal failure

- Circulatory failure

- Coma

(BNF 2007)

\section{Treatment and Prevention}

Treatment is dependent on the seriousness of the toxicity and careful observation is required by the nurse and the individual to recognise symptoms early and to intervene accordingly. A doctor should review the client straight away and blood serum levels should be taken to ascertain the client's condition in order to plan further interventions. No further lithium should be administered to the client and large volumes of intravenous isotonic saline are usually prescribed (Healy 2005). In more severe cases, the client will need to be treated as an emergency and may require haemodialysis (BNF 2007).

Individuals prescribed lithium require information about the drug's actions, the side effects and other specific information required to assist the client to remain within their therapeutic index (Downie et al. 2004). This information should be provided in a manner that is congruent with the clients' ability to understand and verbal information should be reinforced with as much written information as possible. Clients receiving lithium should be encouraged to carry a Lithium card which will explain how to take the drug, what to do if they miss a dose, side effects (including lithium toxicity) and what medications and illnesses alter serum levels. Other information on the lithium card includes:

- Drinking alcohol in small quantities only. 
- Getting advice from nursing, medical or pharmaceutical practitioners before taking other medications and avoiding some medications such as diuretics and non steroid anti inflammatory drugs such as Brufen.

- Drinking plenty of water (especially when hot or during exercise) and not fluctuating daily salt intake.

- If the client is a female, they should consult their medical practitioner before getting pregnant (Downie et al. 2004).

In addition individuals must also be assessed for their motivation to take the drug and to attend for regular serum Lithium levels and thyroid and renal function tests (Prosser et al. 2000). Clients taking lithium should be advised to contact their community mental health nurse, pharmacist or general practitioner if they have any queries or worries about lithium. They should also be aware of the symptoms of lithium toxicity and they should be advised to get medical help if they are feelings unwell, regardless of the cause.

\section{Conclusion}

The administration of medications is an integral part of the mental health nurses roles and in doing so they have a responsibility to be familiar with the preparations that they administer. Imperative to this role is the careful observation of the medication's effects and side effects, as well as educating clients to manage their medication regimes. Knowledge and vigilant observation for serious adverse effects such as the ones discussed in this article is essential if mental health nurses are to recognise, intervene and prevent fatalities that may be caused by a range of medications. 


\section{References}

Birmes P, Coppin, D, Schmitt, L, Lauque D (2003) Serotonin Syndrome: a brief review. CMAJ. 168, 11, 1439-1442.

British Medical Formulary and Royal Pharmaceutical Society of Great Britain (2007) British National Formulary. London, British Medical Formulary and Royal Pharmaceutical Society of Great Britain.

Downie G, MacKenzie J, Williams A (2004) Pharmacology and medicines management for nurses. Edinburgh, Churchill Livinstone.

Galbraith A, Bullock S, Manias E, Hunt B, Richards A. (2007) Fundamentals of Pharmacology: An Applied Approach for Nursing \& Health. London, Prentice Hall.

Healy D (2005) Psychiatric drugs explained. Edinburgh, Churchill Livingstone.

Pelonero AL, Levenson JL, Pandurangi AK (1998) Neuroleptic Malignant Syndrome: A Review. Psychiatric Services. 49, 9, 1163-1172.

Prosser S, Worster B, MacGregor J, Dewar K, Runyard P. Fegan J (2000) Applied pharmacology. London, Harcourt Publishers Limited. 
Rosenfeld G, Loose-Mitchell S (1998) Pharmacology ( $3^{\text {rd }}$ edn). Philadelphia, Lippincott Williams \& Wilkins. 